\title{
COMPETENCIAS DE MAESTROS DE CIENCIAS NATURALES: UNA LECTURA DESDE LAS PRÁCTICAS PEDAGÓGICAS
}

\section{NATURAL SCIENCIES TEACHER COMPETENCIES: A READING FROM PEDAGOGICAL PRACTICES}

\section{Cesar Augusto Hernández Suárez ${ }^{1}$}

\section{Audin Aloiso Gamboa Suárez ${ }^{2}$}

\section{Raúl Prada Núñez ${ }^{3}$}

Colombia

\section{RESUMEN}

El presente artículo de investigación tiene como objetivo analizar los relatos de los docentes que orientan la asignatura de Ciencias Naturales Física en una institución educativa en Norte de Santander, acerca de sus competencias en la práctica pedagógica, en el marco de lo propuesto por el Ministerio de Educación Nacional de

1 Docente investigador de la Universidad Francisco de Paula Santander. Magíster en Enseñanza de las Ciencias por la Universidad Nacional Experimental del Estado Táchira (Venezuela). Correo electrónico: cesaraugusto@ ufps.edu.co, Orcid: orcid.org/0000-0002-7974-5560

2 Docente investigador de la Universidad Francisco de Paula Santander. Doctor en Ciencias de la Educación por la Universidad de Cartagena. Correo electrónico: audingamboa@ufps.edu.co, Orcid: orcid.org/0000-0001-9755-6408

3 Docente investigador de la Universidad Francisco de Paula Santander. Magíster en Ingeniería de Análisis de Datos, Mejora de Procesos y Toma de Decisiones por la Universidad Politécnica de Valencia (España). Correo electrónico: raulprada@ufps.edu.co, Orcid: orcid.org/00000001-6145-1786
Colombia. El enfoque metodológico se centra en el paradigma cualitativo - hermenéutico, utilizando como técnica de recolección de datos la entrevista semiestructurada. Este estudio demostró que las debilidades expresadas por los maestros no se diferencian de otras investigaciones en el mismo campo. Sin embargo, sus discursos se centran en el argumento de la mejora continua lo que a futuro facilitará el desarrollo de su práctica pedagógica.

PALABRAS CLAVE: Prácticas pedagógicas, competencias, saber pedagógico, saber disciplinar.

\section{ABSTRACT}

The objective of this research article is to analyze the accounts of the teachers who guide the Natural Sciences - Physics subject in an educational institution in Norte de Santander, 
about their competences in pedagogical practice, within the framework of what is proposed by the Ministry of National Education of Colombia. The methodological approach focuses on the qualitative - hermeneutic paradigm, using as a data collection technique the semi-structured interview. This study demonstrated that the weaknesses expressed by teachers do not differ from another research in the same field. However, his speeches focus on the argument of continuous improvement, which in the future will facilitate the development of his pedagogical practice.

KEYWORDS: Pedagogical practices, competences, pedagogical knowledge, disciplinary knowledge

\section{INTRODUCCIÓN}

Los vertiginosos cambios relacionados con el desarrollo científico y los avances tecnológicos representan un proceso complejo en el oficio de la enseñanza de la Ciencia desde las prácticas y el saber pedagógico (Díaz, 2008) donde actúan diversos elementos como la didáctica, el currículo, el contexto, los estudiantes y los docentes.

Por otro lado, teniendo en cuenta los cambios que se han generado en las últimas décadas, debido en gran medida al cambio laboral y los retos educativos en el contexto de la globalización: tecno-globalización, la economía informacional y la desregulación de los mercados (Llado, Sanchez y Navarro, 2013), donde surgió el enfoque de la formación por competencias, las políticas de los estados identificadas con la agenda de organismos internacionales como la Organización de las Naciones Unidas para la Educación, la Ciencia y la Cultura (UNESCO), la Organización para la Cooperación y el Desarrollo Económicos (OCDE) y la Organización Internacional para el Trabajo (OIT), entre otros y grupos de expertos, que analizan la exigencia de alinear el sistema educativo formal a las necesidades del sector productivo, hasta la idea de contribuir a la formación de individuos para cubrir un conjunto de necesidades fundamentales, para enfrentar los retos de una sociedad en transformación permanente (Rueda, 2009).

Por eso, los sistemas educativos, incluido el de Colombia, han identificado el desempeño profesional del maestro como un factor determinante del logro de la calidad de la educación, objetivo primordial de los países que están en vía de desarrollo. En estas políticas públicas en educación, incluyen la consolidación de sistemas nacionales de evaluación, y dentro de ellas se ha llevado a cabo la evaluación docente. Pero, se ha realizado de diversas maneras, de acuerdo con la normatividad vigente en cada época, sin que hasta ahora se haya logrado una evaluación de desempeño en forma continua y estructurada que aporte significativamente al mejoramiento de los procesos de aula $y$ al perfeccionamiento profesional. Con la evaluación docente se busca contribuir al mejoramiento de su labor y por consiguiente al mejoramiento de los aprendizajes y desarrollo de los estudiantes, identificando mediante la evaluación de desempeño, las necesidades de desarrollo de conocimientos y competencias propias de la docencia (Ministerio de Educación Nacional, 2003).

Por lo tanto, desde el año 2005, mediante el Decreto Ley 1278 (2002), Estatuto de Profesionalización Docente (los docentes vinculados antes del 2002, se rigen por el Decreto 2277 de 1979), la vinculación a la docencia se establece mediante un proceso de evaluación en las siguientes fases: ingreso, período de prueba, anual de desempeño y de competencias (Ministerio de Educación Nacional, 2009). Es decir, los profesionales que ingresen a la carrera docente vienen presentando una evaluación de ingreso y luego de entrar se someten a un periodo de prueba y 
seguidamente, estando ya en propiedad, dentro de una entidad territorial certificada, inicia su esquema de evaluación de desempeño anual y la evaluación de competencias para ascenso y/o reubicación salarial.

La evaluación de desempeño anual es un proceso permanente que se lleva durante la totalidad del año escolar, que tiene como fin establecer el cumplimiento de las funciones y responsabilidades por parte del docente, por medio de competencias funcionales como el dominio curricular, planeación y organización académica, pedagógica y didáctica y evaluación del aprendizaje para la gestión académica entre otras (Marquez, Vergel y Gallardo, 2020). Están competencias se evalúan junto con las comportamentales. Por otro lado, está la evaluación de competencias para el ascenso o reubicación de nivel lo cuál evalúa las competencias disciplinares, pedagógicas y comportamentales. Hasta el 2014, se aplicaba de manera escrita, pero desde el 2015, se cambió hacia una evaluación de carácter diagnóstico formativo mediante la presentación de grabaciones de videos.

Además, las prácticas pedagógicas de los docentes de Ciencias Naturales, particularmente los de Física, deben ser pensadas y repensadas en las intervenciones pedagógicas porque "la enseñanza de las ciencias tradicional ha buscado el fomento de una actitud científica a la vez que se contrapone a una actitud científica nata referente a la curiosidad natural del ser humano" (Santos, 2014, p. 44). Además, argumenta que los profesores en el aula siguen siendo, el que explica y, los que definen lo que deben de hacer los discentes, es decir, son los estudiantes los que deben escuchar y copiar.

De allí, que la práctica pedagógica requiere competencias que son esenciales en el quehacer docente. Según Gallardo, Hernández y Arévalo (2017) la "competencia adecuado al campo de la educación significa un saber en contexto" (p. 15) para ofrecer una enseñanza de calidad, reto que se han de afrontar entre los actores del hecho educativo con metodologías pedagógicas y didácticas en su deber ser. Donde se requiere de acuerdo con Tamayo (2017), de "un análisis de la realidad social de las prácticas pedagógicas, entendida desde sus diferentes dimensiones" (p. 3).

De este modo, se trata de analizar las opiniones de los docentes que orientan Ciencias Naturales - Física acerca de sus competencias en la práctica pedagógica, el marco de lo propuesto por el Ministerio de Educación Nacional de Colombia, que permitan la reflexión acerca de su acción para impulsar cambios orientados a la mejora de los procesos educativos de los que son responsables.

\section{LA PRÁCTICA PEDAGÓGICA}

En Colombia, la historia de la realidad de la relación estudiante-docente-escuela-currículo, ha sido y es vista desde otra perspectiva por pedagogos con aspectos más científicos y complejos desde lo metodológico. Al respecto, Zuluaga (1999, p. 10) señala que "la pedagogía no es sólo un discurso acerca de la enseñanza, sino también una práctica cuyo campo de aplicación es el discurso, ... la pedagogía es discurso y práctica". Para esta autora y el grupo de "Historia de las prácticas pedagógicas en Colombia", fue necesario crear la noción de práctica pedagógica con el fin de historiar la pedagogía en el país.

Por otro lado, Martínez (2012), señala que el concepto de práctica pedagógica implica la relación de tres elementos: el maestro, el saber y la escuela; además, explica que no sólo se refiere a la acción del maestro enseñante y la enseñanza, sino que también involucra la realidad institucional de la escuela con todos los elementos y sujetos que la dinamizan a través de saberes pedagógicos (Touriñán, 2020). 
Las prácticas pedagógicas planteadas en el marco del constructivismo y potenciadas por el diseño curricular por competencias, plantean el desarrollo de los proyectos de aula, que permiten que los propios estudiantes, bajo la guía del profesor, desplieguen un conjunto de actividades a través de las cuales infieren y construyen conocimiento que deben aprender de acuerdo con el plan de estudios o diseño curricular (Salamanca y Hernández, 2018)

Luego, la práctica pedagógica en la educación es la aplicación, articulación, y actuación directa de los participantes en el proceso educativo (docentes, estudiantes, institución y el cuerpo de conocimientos), haciendo énfasis en el proceso de enseñanza y aprendizaje que se desarrolla en un contexto determinado.

Por tanto, pensar la práctica pedagógica hoy, es una tarea muy compleja que no se puede eludir, es un reto que involucra a la escuela, su entorno y la sociedad con todas sus complejidades culturales, económicas y políticas. Es un reto que implica un esfuerzo sobrehumano en distintas y difíciles direcciones, que involucran la escuela, su entorno y la sociedad con todas sus complejidades culturales, económicas y políticas. $\mathrm{Y}$, esto elegimos y debemos asumirlo con responsabilidad ética y moral.

\section{COMPETENCIAS DOCENTES}

En el ámbito de las competencias Tobón (2005), afirma que las competencias poseen diversas acepciones, y que tiene un origen latino en relación con el verbo competir, y con el sustantivo competencia que equivale a ser capaz o ser apto o adecuado. Este autor, propone conceptuar las competencias como "procesos complejos que las personas ponen en acción-actuacióncreación para resolver problemas, aportando a la construcción y transformación de la realidad, integrando el saber ser, el saber hacer y el saber conocer, teniendo en cuenta los requerimientos del entorno" (p. 44).
Ahora, para Hernández, Pabón y Prada (2017), el concepto de "competencia ha alcanzado gran relevancia en el contexto educativo contemporáneo porque la escuela ha dejado de ser la fuente fundamental del saber. Los estudiantes están rodeados de gran cantidad de información, que les llega por distintas y numerosas vías" (p. 196).

De este modo, diseñar planes de estudio por competencias desarrollarán las capacidades requeridas en las personas y estas se puden referir como un "conjunto de conocimientos, habilidades, actitudes, comprensiones y disposiciones cognitivas, socioafectivas y psicomotoras apropiadamente relacionadas entre sí para facilitar el desempeño flexible, eficaz y con sentido de una actividad en contextos relativamente nuevos y retadores" (Ministerio de Educación Nacional, 2006, p. 49).

Para Perrenoud (2005) el docente debe tener otras características diferentes de las que usualmente tiene, debido a los cambios que la sociedad demanda y en especial a la naturaleza de los seres humanos y destacando que es una labor muy difícil; por tanto, las competencias, que debe emplear un docente en los contextos escolares y sociales actuales, deben:

Organizar y animar situaciones de aprendizaje; Gestionar la progresión de los aprendizajes; Elaborar y hacer evolucionar dispositivos de diferenciación; Implicar a los alumnos en sus aprendizajes y en su trabajo; Trabajar en equipo; Participar en la gestión de la escuela; Informar e implicar a los padres; Utilizar las nuevas tecnologías; Afrontar los deberes y los dilemas éticos de la profesión; Organizar la propia formación continua (Perrenoud 2005, p. 6). 
Es decir, se alcanza a percibir en este concepto, que el autor hace hincapié en los componentes pedagógico y comportamental, pero desde el punto de vista de la gestión estratégica. Igualmente, Beneitone et al, (2007), señalan que se producen 27 competencias importantes para el área de la educación, las cuales corresponden a los aspectos: disciplinar, pedagógico de gestión y científico.

Asimismo, la labor educativa exige actualmente que los docentes desarrollen múltiples competencias vinculadas con la capacidad de diseñar experiencias de aprendizaje significativas, en las que los estudiantes sean el centro del proceso de enseñanzaaprendizaje (Mendoza, Hernández y Prada). En este contexto, las competencias docentes son entendidas como el conjunto de conocimientos, habilidades (destrezas) y actitudes (motivación, valores éticos, emociones, intereses, rasgos de personalidad y otros componentes sociales y de comportamiento) que necesitan los profesionales de la educación para resolver de forma satisfactoria las situaciones a las que se enfrentan en su práctica pedagógica (Hernández, Gamboa y Ayala, 2014; Hernández, Arévalo y Gamboa, 2016).

Igualmente, el Ministerio de Educación Nacional (2008) define una competencia (del docente) como una característica intrínseca de un individuo (docente) que se manifiesta en su desempeño particular en contextos determinados (práctica pedagógica), cuando es capaz de resolver con éxito diferentes situaciones de forma flexible y creativa que involucra la interacción de disposiciones (valores, actitudes, motivaciones, intereses, rasgos de personalidad, entre otros), conocimientos y habilidades.

Concretamente, para el caso de la labor docente en Colombia, el Decreto-Ley 1278 (2002) define una competencia (del docente) como una característica subyacente en una persona (docente) causalmente relacionada con su desempeño y actuación exitosa en un puesto de trabajo (práctica pedagógica) y señala también que su evaluación debe permitir la valoración del logro y acción, ayuda y servicio, influencia, liderazgo y dirección, cognitivas y eficacia personal.

Asimismo, Ministerio de Educación Nacional, en la Resolución 09317 (2016), y en la guía metodológica evaluación anual de desempeño laboral se señala las competencias para los docentes como funcionales y comportamentales. Las funcionales, que se expresan en las actuaciones intencionales del docente en diferentes áreas de la gestión institucional, en el proceso de enseñanza-aprendizaje e implica las acciones de: planeación, ejecución, evaluación y articulación con el entorno social que se encuentra relacionado a la labor docente. La comportamental tiene que ver con las actitudes, los valores, los intereses, las motivaciones y las características de personalidad. Estas competencias, para el docente, se evidencian en el desarrollo de las áreas de gestión académica, administrativa y comunitaria y en la autoevaluación institucional (Ministerio de Educación Nacional, 2008).

Por tanto, teniendo en cuenta la necesidad de contar con criterios que permitan determinar el nivel de desempeño de los docentes se hace necesario definir un perfil que enuncie las competencias para tener éxito en el desarrollo de su ejercicio profesional. Es por eso, que el perfil docente debe reunir un conjunto de competencias organizadas por unidades, requeridas para realizar la actividad docente, de acuerdo con criterios valorativos y parámetros de calidad (Hernández, Pabón y Prada, 2017).

Por otra parte, el Ministerio de Educación Nacional (2006), define una competencia como saber hacer en situaciones concretas que requieran la aplicación creativa, flexible y responsable de conocimientos, habilidades y 
actitudes. Responde al ámbito del saber qué, saber cómo, saber por qué y saber para qué.

Esta definición se refiere a las competencias que debe desarrollar un estudiante, pero también aplica para el caso del docente. En este caso, algunas están relacionadas con los saberes y conocimientos disciplinares. Otras son independientes del saber y se relacionan con el comportamiento, con estrategias que emplean para desarrollar su trabajo de la mejor manera, o con atributos personales que favorecen la ejecución de una actividad. En efecto, el ejercicio docente requiere de competencias que involucra el saber disciplinar (en este caso, la Física), pedagógico y comportamental.

\section{METODOLOGÍA}

La presente investigación se aborda desde el paradigma cualitativo - Hermenéutico, puesto que pretende encontrar desde el mismo docente, respuestas a preguntas pertinentes de la realidad de su práctica pedagógica y de este modo identificar un tipo de conocimiento que se construye en el diálogo, la interacción, la intersubjetividad y la cotidianidad (Gamboa, Lago y Hernández, 2016). Por otra parte, la hermenéutica se considera como

(...) esta alternativa es una opción que no se agota exclusivamente a una dimensión filosófica, sino que trasciende a una propuesta metodológica de comprensión de la realidad social bajo la metáfora de un texto susceptible de ser interpretado y para el efecto desarrolla en sus distintas acepciones y caminos metodológicos con particularidades muy propias que la hacen distinta a otras alternativas de investigación. (Sandoval, 1997, p. 60)

En la versión de Ricoeur (1987), la hermenéutica clásica se define como la teoría de las reglas que gobiernan una exégesis, es decir, una interpretación de un texto particular o una colección de signos susceptible de ser considerada como un texto. En esta perspectiva, uno de los conceptos básicos es el de "círculo hermenéutico" que describe el movimiento entre la forma de ser del intérprete y el ser que es revelado por el texto.

Los sujetos seleccionados fueron considerados informantes clave; a juicio de Taylor y Bogdan (1987) son conocedoras del objeto y contexto de estudio que "...los muestran, los presentan a otros, responden por ellos, les dicen cómo deben actuar y le hacen saber cómo son vistos por otros" (p. 61). Entonces, Los sujetos de investigación corresponden a cinco docentes que enseñan la Física en una institución educativa pública de la ciudad de Cúcuta, Colombia (ver tabla 1). 
Tabla 1

Caracterización de los informantes clave (docentes)

\begin{tabular}{|c|c|c|c|}
\hline Sujeto & Sexo & Pregrado / Postgrado & $\begin{array}{c}\text { Escalafón/ } \\
\text { decreto }\end{array}$ \\
\hline $\begin{array}{c}\text { Docente } \\
\mathbf{1}\end{array}$ & $\mathrm{F}$ & Lic. Biología y Química / Educación y gestión ambiental & $14 / 2277$ \\
\hline $\begin{array}{c}\text { Docente } \\
\mathbf{2}\end{array}$ & $\mathrm{M}$ & Ing. Electrónico / Administración de la informática & educativa \\
\hline $\begin{array}{c}\text { Docente } \\
\mathbf{3}\end{array}$ & $\mathrm{F}$ & Lic. Matemáticas y Física & $2 \mathrm{~A} / 1278$ \\
\hline $\begin{array}{c}\text { Docente } \\
\mathbf{4}\end{array}$ & $\mathrm{M}$ & Lic. Matemáticas y Computación & $13 / 2277$ \\
\hline $\begin{array}{c}\text { Docente } \\
\mathbf{5}\end{array}$ & $\mathrm{M}$ & Ing. Mecánico & $2 \mathrm{~A} / 1278$ \\
\hline
\end{tabular}

Fuente: Elaboración propia

Se realizaron entrevistas semiestructuradas de 11 preguntas relacionadas con las competencias docentes aplicadas en sus prácticas pedagógicas y la opinión que tienen de ellas. El instrumento se construyó teniendo en cuenta los documentos de evaluación de competencias para el ascenso o reubicación de nivel salarial en el escalafón de profesionalización docente de los docentes y directivos docentes regidos por el Decreto Ley 1278 de 2002 y la Guía 31: Guía metodológica para la evaluación anual de desempeño laboral de los docentes de Colombia regidos por el Decreto Ley 1278 de 2002. De este modo, cada una de las preguntas están en correspondencia con las dimensiones de las competencias docentes sugeridas en estos documentos.

\section{RESULTADOS Y DISCUSIÓN}

\section{Currículo y su relación con las competencias en la Prácticas pedagógicas de los docentes}

Desde el punto de vista de los docentes, en la categoría currículo se evidencian dos subcategorías, fundamentos curriculares y currículo por competencias. Con respecto a los fundamentos curriculares, los docentes reconocen en el currículo elementos fundamentales como los estándares básicos, la planeación, los contenidos y los proyectos pedagógicos. También, lo conciben como normas o lineamientos, como el contenido que se enseñan a los estudiantes, como un documento de planes y proyectos y con todo lo relacionado con lo institucional y académico, que está direccionado por el Ministerio de Educación Nacional en el marco de las leyes educativas y que configuran elementos como las competencias, los desempeños, los contenidos y los métodos.

Es importante precisar, que el currículo se expresa en el Proyecto Educativo Institucional y se hace realidad en las aulas de clase en la interacción docente - estudiante - saber - escuela (Martínez, 2012) es decir, en la práctica pedagógica (Zuluaga, 1999). Además, es un constructo que permite dinamizar las acciones $\mathrm{o}$ prácticas pedagógicas, plasmadas en un documento que representa e identifican la acción social institucional de la realidad donde está inmersa (Luna y López, 2011).

Además, de acuerdo con lo afirmado por los docentes se forman grupos de conceptos sobre estándares básicos, planeación académica y contextualizada y proyectos pedagógicos. Lo cual evidencia la relación que ellos encuentran 
entre esos conceptos y los fundamentos curriculares (planeamiento curricular). Aquí surgieron dos posturas, la primera, relacionada con la planificación académica centrada en la práctica pedagógica y en las actividades del aula, en la que destacan situaciones que tienen que ver con la definición de objetivos, momentos didácticos y estrategias de evaluación y retroalimentación, mientras que la segunda referencia la vinculación en la enseñanza de las Ciencias y la conexión del contexto con la enseñanza basada en una planificación contextualiza, basada en la realidad y en constante referencia a situaciones cotidianas como porque todo lo que tiene que ver con la Física es lo que nos rodea (Prada, Hernández y Ramírez, 2020). Otro elemento importante es el relacionado con la formulación de proyectos pedagógicos como un elemento básico de la planificación y la utilización de los estándares y lineamientos curriculares como ejes rectores de todas las actividades académicas.

Tal como lo proponen Luna y López (2011), el currículo identifica y explicita el PEI, y le corresponde al docente, diseñarlo teniendo en cuenta la realidad de la escuela, ya que allí están plasmado, la identidad y los alcances institucionales. También es función del docente, hacerlo realidad, y, además, realizar el debido seguimiento para evaluar la pertinencia, la validez, la eficacia, la efectividad de dicho currículo. Además, la guías y lineamientos del Ministerio de Educación Nacional, establece los parámetros de la evaluación anual de desempeño, en el se describen los criterios que debe tener un docente para evidenciar el trabajo de organización escolar destacando las funciones de planeación, ejecución y evaluación de las diferentes actividades curriculares en función de los proyectos institucionales y considerando el contexto de los estudiantes (Veloza y Hernández, 2018).
Por otra parte, en la subcategoría "currículo por competencias" se evidencia la relación entre "concepto de competencias", "modelo por competencias" y "desempeño laboral". En ella, los docentes consideran que dentro de sus funciones está desarrollar en los estudiantes las habilidades necesarias para hacer de ellos personas competentes. Pero, los docentes solo las entienden en sentido general, como un saber, un saber hacer y un saber ser (concepto de competencias), una visión más estrecha y limitada, pero muy cercano al concepto de autores como Tobón (2005), Beneitone et al. (2007), e inclusive del mismo Ministerio de Educación Nacional (2006), entre otros, que se queda en el que, pero que no trasciende a el para qué, donde y como (López, 2016). En este orden de ideas, una persona es competente cuando es capaz de solucionar problemas de situaciones reales en forma eficaz, efectiva y eficiente, y "optar por una educación en competencias representa la búsqueda de estrategias de enseñanza que sitúen su objeto de estudio en la forma de dar respuesta satisfactoria a situaciones reales y, por lo tanto, complejas" (Zabala y Arnau, 2007, p. 42).

En cuanto a lo relacionado con modelo por competencias los docentes señalan que es una estrategia del Ministerio de Educación Nacional, para que los estudiantes puedan adaptarse o vincularse con el entorno, al mundo laboral. Además, la formación por competencias está relacionada con el hecho de aplicar para sus procesos de enseñanza actividades muy relacionadas con el entorno, situaciones de la vida real o del contexto local, regional y global. También, resaltan el hecho de que la ciencia física es la explicación de todo lo que nos rodea y puede contribuir a que el estudiante comprenda más su entorno. Finalmente, para ellos existe una interacción muy importante y estratégica entre currículo, competencias y contenidos y que todo esto está direccionado desde el Ministerio de Educación Nacional. 
Lo anterior deja ver las brechas entre lo que piensan los docentes y el concepto de modelo curricular basado en competencias donde se concibe como una estructura que integra acciones, objetivos, operaciones, contenidos, recursos, metodologías y procedimientos articulados al contexto de un proyecto educativo, es decir, a un currículum guiado por las competencias (López-Goñi y Goñi, 2015).

Para finalizar, en lo manifestado por los profesores, también se evidencia que al currículo por competencias le asocian las competencias para el desempeño laboral, es decir, tal como lo afirman Escobar (2005) y Trujillo-Segoviano (2014), quienes señalan que está asociado al contexto empresarial, para designar un conjunto de elementos o factores relacionados con el éxito y el desempeño de las personas, ya que consideran que la formación por competencias tienen un objetivo mercantilista, que al final sólo beneficiaría a los poderes económicos, una noción muy asociada al neoliberalismo (Miñana y Rodríguez, 2003; Trujillo, 2009), y la globalización (Lladó, Sánchez y Navarro, 2013; Trujillo-Segoviano, 2014 ).

En general, en la categoría currículo, los discursos de los profesores difieren del amplio espectro conceptual tanto de currículo como de competencia y sus derivaciones. En todos los casos reconocen que la esencia conceptual se fundamenta en los contenidos y los parámetros dados por el Ministerio de Educación (por ejemplo, con respecto a los estándares), y según ellos, brinda meras capacitaciones, sin tener construcciones epistemológicas bien constituidas quedándose en nociones básicas, demostrando una concepción conceptual genérica. Por eso para abordar los conceptos desde esta epistemología es necesario tener en cuenta sus múltiples dimensiones y ejes de significación (Bacarat y Graziano, 2002), esto requiere de un análisis crítico y reflexivo para comprenderlo y usarlo.
Se entiende, que lo analizado anteriormente, puede afectar el actuar del docente (su práctica pedagógica), por eso en cierta medida se podría explicar que a pesar de tener buenas valoraciones en las evaluaciones del desempeño anual muchos docentes no aprueban (Ministerio de Educación Nacional, 2014b, Gallego y Vásquez, 2015). Lo anterior, de cierto modo, también podría estar relacionado con el bajo logro de los estudiantes, como lo evidencian (Schwerdt \& Wuppermann, 2011; Echazarra, Salinas, Méndez, Denis, \& Rech, 2016; Le Donné, Fraser \& Bousquet, 2016; Cordero, y Gil-Izquierdo, 2018), generalmente centradas en las estrategias del docente, pero sigue siendo incierto lo que realmente determina una enseñanza efectiva.

También es importante señalar, por ejemplo, que el currículo tiene varios matices, en especial, el que se refiere a los procesos de reflexión que enrutan una transformación profunda de aspectos como el cambio en las prácticas pedagógicas o las nuevas formas de enseñabilidad de las ciencias que confiere la didáctica (Díaz, 2014). Otro concepto importante que no se hace visible en los relatos de los maestros, es la construcción y deconstrucción curricular para comprender la descomposición de las estructuras conceptuales de la arquitectura de la institución educativa (Gamboa, Hernández y Montes, 2018).

Con respecto al concepto competencias, a pesar de que es polisémico y complejo, los docentes tienen una visión reduccionista e instrumental, inspirada en una perspectiva cognitivo-conductual (Moreno, 2012), enfocado únicamente en la formación laboral que en el desarrollo integral de la persona. Es necesario, precisar que una competencia es más que conocimientos, destrezas y actitudes, involucra la habilidad de enfrentar demandas complejas (concepto asociado al proyecto DeSeCo citado por Hernández, Ayala y Gamboa, 2014). Esto podría deberse a que la noción de competencias 
que se emplea en educación no proviene de un único paradigma teórico, sino de múltiples fuentes (psicología, lingüística, socióloga, educación para el trabajo y filosofía) propuesto por Zubiría, (2002) y se ha aplicado a otras áreas de una forma asistemática y desconexa (Tobón, 2005). Por lo que este concepto puede ser asumido como un saber hacer razonado para hacer frente a la incertidumbre en un mundo cambiante en lo social, político y laboral dentro de una sociedad globalizada y en continuo cambio, no como comportamientos observables, sino como una compleja estructura de atributos necesarios para desempeñar en determinadas situaciones donde se combinan conocimiento, actitudes, valores, habilidades y tareas.

Por ello, al asumir la complejidad de las competencias, implica reconocer que son un enfoque inacabado $y$ en constante construcción-deconstrucción-reconstrucción requiriéndose continuamente del análisis crítico y la autorreflexión para comprenderlo y usarlo (Tobón, 2005).

\section{Categoría práctica pedagógica y su relación con las competencias}

En la categoría práctica pedagógica se evidencian principalmente las subcategorías didáctica y evaluación.

Los discursos de los docentes se centran principalmente en dos aspectos: el primero de ellos es el académico, donde según sus relatos se enfocan en enseñar todo lo relacionando con las teorías y conceptos de la Física, manifiestan tener un buen dominio disciplinar y didáctico. Para los docentes del Decreto 1278 de 2002, entienden que este es un aspecto que se evalúa (Didáctica de la Física), que aplican al contexto del estudiante y situaciones reales mediante el uso enseñanza para la comprensión (que lo identifican como el modelo pedagógico de la institución), pero también experimentos, solución de problemas, enseñanza contextualizada, enfoque constructivista, aprendizaje significativo, actividades de forma individual y grupal, actividades de carácter investigativo y hasta clases magistrales tradicionales (estrategias didácticas). En lo metodológico señalan que aplican tanto el método inductivo como el deductivo y el método científico, eso siempre enfocado y planeado de acuerdo con las normas institucionales y el proyecto educativo de la institución.

De acuerdo con lo anterior, la estrategia pedagógica plasmado en el PEI de la institución es la enseñanza para la comprensión (Perkins, 1999), el cual los docentes dicen emplear, junto con otras estrategias, pero al hacer un análisis no se logra evidenciar este enfoque en la planeación escolar.

Además, el no tener claridad en los elementos del PEI y en sus principios filosóficos, conlleva a que y estos dejan de responder a las situaciones y necesidades de los estudiantes y la comunidad. Luego ese PEI no es pertinente y no logra la formación integral del educando. Ahora, lo que preocupa es el seguimiento que se hace a estos procesos (esto se evidencia en que los docentes poco lo mencionan y cuando lo hacen no son precisos, (a pesar de manifestar tener claridad en esta etapa) porque no se logran identificar las debilidades por parte de los docentes, directivos y comunidad educativa, por ejemplo, en la evaluación de desempeño de los docentes y la autoevaluación institucional afecta la calidad y gestión, es decir se desconoce la realidad institucional. Por lo que se hace necesario hacer una resignificación del PEI para aportar a la transformación curricular a partir de una estrategia pedagógica propia de la institución (Hernández, 2018).

Lo anterior, no se puede contrastar con lo propuesto por el Ministerio de Educación Nacional donde el PEl y el plan de mejoramiento son herramientas para orientar las acciones escolares hacia el logro de las metas 
propuestas. Es en la institución educativa donde se materializan el PEI, el currículo, y se concretan la planeación curricular, las actividades pedagógicas, las evaluaciones y autoevaluaciones y las relaciones de los miembros de la comunidad educativa. (Ministerio de Educación Nacional, 2008b).

El fenómeno analizado y los hallazgos del estudio limitan el desarrollo institucional y las posibles acciones para su solución. Por eso es necesario la cultura de autoconocimiento e integrar la autoevaluación con fines de mejora continua al sistema de gestión institucional (Peña, Almuiñas y Galarza, 2018) y esta responsabilidad recae sobre las personas encargadas de la gestión escolar, es decir docentes y directivos (Romero, 2004).

La subcategoría evaluación presenta dimensiones como la planeación, ejecución y el seguimiento. En sus relatos, los docentes dicen que hay diversas clases de evaluación, tales como: cuantitativa y cualitativa; hablan de evaluación contextualizada como un tipo de evaluación; además que evalúan lo cognitivo, lo procedimental y lo actitudinal, es decir, evaluar al estudiante integralmente. También afirman establecer la diferencia entre evaluación por contenidos y por competencias, pero se evidencia un fuerte énfasis en lo memorístico, a pesar de que algunos consideran importante el enfoque por competencias, lo más cercano a esta, es lo mencionado como resolución de problemas, no se encuentran evidencias específicas sobre esta.

Lo anterior se puede explicar de la siguiente manera: por un lado, la dificultad del docente para entender el concepto de competencia, además de carecer de la experiencia sobre la aplicación práctica de un currículo basado en competencias. $\mathrm{Y}$ por el otro, la dificultad propia de la evaluación del aprendizaje, pues están dadas las condiciones de ambigüedad y desconcierto que se ve los centros educativos (Arribas, 2017), tal como sucede en este caso.

Lo anterior deja en evidencia lo expresado por Moreno (2012), quien afirma que la evaluación educativa que predomina corresponde a visión estrecha, caracterizada por un afán excesivo por medir los productos de aprendizaje descuidando los procesos y que por lo general mide los contenidos de corte cognoscitivo, se centra en el profesor, emplea solo pruebas escritas, no considera los distintos ritmos y estilos de aprendizaje, entre otros y que va en contravía de la formación por competencias, que demanda de la participación y comprometida del estudiante con su propio aprendizaje. Nadie puede adquirir las competencias por otro.

En los hallazgos encontrados en la opinión de los docentes se evidencia un enfoque de evaluación sumativa y no señalan por ejemplo la utilización de la evaluación formativa, también afirman que la evaluación es un indicador para el profesor, de qué tanto está asimilando o está reteniendo el estudiante, luego no hay retroalimentación solo recuperaciones. Así mismo, en los relatos de los maestros se demuestra que no existe un procedimiento formal para evaluar y hacer seguimiento de esta. Esto también podría deberse a lo abordado en el apartado anterior, al no haber claridad sobre el enfoque pedagógico de la institución y a la identidad que lo relacione con este.

Hay investigaciones que evidencian la oposición a la evaluación formativa y la preferencia por la evaluación sumativa debido a las dificultades que trae para los docentes la transformación de su práctica pedagógica (Hernández, Prada y Gamboa, 2019), en particular en cuanto a la adopción del enfoque formativo de la evaluación (Martínez, 2013) y donde la retroalimentación es la clave para una evaluación orientada al aprendizaje (Canabal y Margalef, 2017), así como su importancia para las Ciencias Naturales (Talanquer, 2015) 
Finalmente, la evaluación en el proceso enseñanza - aprendizaje debe ser continua, permanente y formativa. Debe servir para verificar permanentemente los avances y las dificultades de los estudiantes en relación con las metas de aprendizaje planeadas.

De igual manera la evaluación debe proveer al docente herramientas para replantear las estrategias tanto metodológicas de la enseñanza como evaluativas, buscando ayudar a los estudiantes (Ministerio de Educación Nacional, 2017; Moreno, 2016).

\section{CONCLUSIONES}

Este estudio demostró que las debilidades expresadas por los docentes no se diferencian de otras investigaciones en el campo. Sin embargo, lo positivo es que los docentes manifiestan el interés de mejorar su práctica pedagógica en todos los aspectos que ella contempla.

Dentro de los elementos por mejorar, los docentes manifiestan que deben usar diversas estrategias para apoyar sus prácticas pedagógicas y que desconocen los elementos teóricos y metodológicos del enfoque pedagógico propuesto en el proyecto educativo institucional.

Así mismo, se logró evidenciar que hay aspectos que develan prácticas pedagógicas tradicionales que difieren de la formación por competencias. También, existen divergencias entre lo disciplinar y pedagógico, pues el mostrar un dominio teórico conceptual en lo concerniente con la física no implica que también lo sea en el campo pedagógico. Es importante señalar que la relación saber disciplinar y saber pedagógico en la formación del maestro es un elemento clave para el éxito en la práctica pedagógica.

\section{REFERENCIAS BIBLIOGRÁFICAS}

Arribas, J. (2017). La evaluación de los aprendizajes. Problemas y soluciones. Profesorado. Revista de Currículum y Formación de Profesorado, 21(4), 381-404.

Bacarat, M. \& Graziano, N. (2002). ¿Sabemos de qué hablamos cuando usamos el término competencia? En G. Bustamante, S. Zubiría, M. Bacarat, N. Graziano, L. Marín, J. Gómez y E. Serrano (Eds.), El concepto de competencia II (pp. 63-89). Bogotá: Sociedad Colombiana de Pedagogía.

Beneitone, P., Esquetini, C., González, J., Marty, M., Siufi, G. \& Wagenaar, R. (Eds). (2007). Reflexiones y perspectivas de la educación superior en América Latina: informe final, proyecto Tuning América Latina 2004-2007. Bilbao, España: Publicaciones Universidad de Deusto.

Canabal, C. \& Margalef, L. (2017). La retroalimentación: la clave para una evaluación orientada al aprendizaje. Profesorado, Revista de Currículum y Formación del Profesorado, 21(2), 149-170. Recuperado de https://recyt.fecyt.es/ index.php/profesorado/article/view/59454

Cordero, J. M., \& Gil-Izquierdo, M. (2018). The effect of teaching strategies on student achievement: An analysis using TALISPISA-link. Journal of Policy Modeling, 40(6), 1313-1331. DOI: https://doi.org/10.1016/j. jpolmod.2018.04.003

De Zubiría, S. (2002). La mala pedagogía se hace con buenas intenciones. En G. Bustamante, S. Zubiría, M. Bacarat, N. Graziano, L. Marín, J. Gómez y E. Serrano (Eds.), El concepto de competencia II. Bogotá: Sociedad Colombiana de Pedagogía. 
Díaz, V. (2008) ¿Cómo formar un docente investigador? Educare, 12(1), 3-16. Recuperado de http://revistas.upel.edu.ve/index.php/ educare/article/view/40/39

Díaz-Barriga, Á. (2014). Construcción de programas de estudio en la perspectiva del enfoque de desarrollo de competencias. Perfiles educativos, 36(143), 142-162. Recuperado de https://www.iisue.unam.mx/perfiles/ articulo/2014-143-construccion-de-programasde-estudio-en-la-perspectiva-del-enfoque-dedesarrollo-de-competencias.pdf

Echazarra, A., Salinas, D., Méndez, I., Denis, V. \& Rech, G. (2016). How teachers teach and students learn: Successful strategies for school. OECD Education Working Papers, 130. Paris: OECD Publishing, https://doi. org/10.1787/5jm29kpt0xxx-en

Escobar, M. (2005). Las competencias laborales: ¿La estrategia laboral para la competitividad de las organizaciones? Estudios Gerenciales, 20(96), 31-56. Recuperado de https://www.icesi.edu.co/revistas/index.php/ estudios_gerenciales/article/view/170

Gallardo, H., Hernández, C. \& Arévalo, M. (2017). Enseñanza de las ciencias. Una década de investigación en la maestría en práctica pedagógica. Ecoe Ediciones Ltda.

Gallego, A. \& Vásquez, O. (2015). Pruebas de ascenso y reubicación salarial en maestros. Un problema de dignificación de la labor docente Título. Revista Virtual Universidad Católica del Norte, 46, 60-70. Recuperado de http://revistavirtual.ucn.edu.co/index.php/ RevistaUCN/article/view/699/1226

Gamboa, A., Hernández, C. \& Montes, A. (2018). Currículo y enfoque pedagógico: imaginarios institucionales sobre docencia en una universidad pública en Norte de Santander. Revista Colombiana de Ciencias Sociales, 9(1), 46-59. DOI: https://doi.org/10.21501/22161201.2608
Gamboa, A., Lago, D. \& Hernández, F. (2016). Calidad de la docencia universitaria comprensión de los discursos y políticas institucionales de una universidad pública en norte de Santander. Saber Ciencia y Libertad, 11(1), 197-210. DOI: http://dx.doi.org/10.22525/ sabcliber.2016v11n1.197210

Gómez, J. M. R. (1995). El maestro y las instituciones educativas. Ensayos: Revista de la Facultad de Educación de Albacete, (10), 171182. Recuperado de https://dialnet.unirioja.es/ descarga/articulo/2282557.pdf

Hernández, C. (2018). Fortalecimiento de competencias científicas: La investigación como estratégica pedagógica. Horizontes Pedagógicos, 19(2), 91-100. DOI: https://doi. org/10.33881/0123-8264.hop.19205

Hernández, C., Arévalo, M., \& Gamboa, A. (2016). Competencias TIC para el desarrollo profesional docente en educación básica. Praxis \& Saber, 7(14), 41 - 69. https://doi. org/10.19053/22160159.5217

Hernández, C., Gamboa, A. \& Ayala, E. (2014). Competencias TIC para los docentes de Educación superior. En J. Asenjo, Ó. Macías y J. C. Toscano (Coords.), Congreso Iberoamericano de Ciencia, Tecnología, Innovación y Educación. Buenos Aires, Argentina: Organización de Estados Iberoamericanos para la Educación, la Ciencia y la Cultura. Recuperado de http://www. oei.es/congreso2014/memoriactei/837.pdf

Hernández, C., Prada, R. \& Gamboa, A. (2019). Conceptions and pedagogical practices on the mathematical processes of teachers. Journal of Physics: Conference Series, 1408, 012009. DOI: http://dx.doi.org/10.1088/17426596/1408/1/012009

Hernández-Suárez, C., Pabón-Galán, C. \& Prada-Núñez, R. (2017). Desarrollo de competencias y su relación con el contexto educativo entre docentes de ciencias naturales. 
Revista Virtual Universidad Católica del Norte, 51, 194-215. Recuperado de http://revistavirtual. ucn.edu.co/index.php/RevistaUCN/article/ view/852/1370

Le Donné, N., Fraser, P., \& Bousquet, G. (2016), Teaching Strategies for Instructional Quality: Insights from the TALIS-PISA Link Data. OECD Education Working Papers, 148, Paris: OECD Publishing. DOI: https://doi. org/10.1787/5jln1hlsrOlr-en

López, E. (2016). En torno al concepto de competencia: un análisis de fuentes. Profesorado. Revista de Currículum y Formación del Profesorado, 20(1), 311-322 https:// recyt.fecyt.es/index.php/profesorado/article/ view/49881

López-Goñi, I. \& Goñi, J. M. (2015): Hacia un currículum guiado por las competencias. Propuestas para la acción. Pamplona: Servicio Editorial de la Universidad Pública de Navarra.

Luna, E. \& López, G. (2011). EL Currículo: concepciones, enfoques y diseño. Revista UNIMAR, 29(2), 65-76. Recuperado a partir de http://editorial.umariana.edu.co/revistas/index. php/unimar/article/view/217

Llado, D., Sánchez, L., \& Navarro, M. (2013). Competencias profesionales y empleabilidad en el contexto de la flexibilidad laboral. México: Palibrio LLC

Martínez, A. (2012). Práctica pedagógica: historia y presente de un concepto. D. Barragán, A. Gamboa y J. Urbina (Comp.). Práctica pedagógica: Perspectivas teóricas, (pp. 56-68). Bogotá: Ecoe ediciones.

Mendoza, S., Hernández, C. \& Prada, R. (2019). Principios de la argumentación y las prácticas argumentativas en la formación de docentes en matemáticas. Bogotá: Ecoe Ediciones Universidad Francisco de Paula Santander.
Márquez Peñaranda JF., Vergel Ortega M. \& Gallardo Pérez H de J. (2020). Percepción en la planificación de cursos de física: relaciones en entornos sincrónicos. Revista Boletín Redipe, 9(12), 93-100. Recuperado de: https://revista. redipe.org/index.php/1/article/view/1137

Martínez, F. (2013). Dificultades para implementar la evaluación formativa: Revisión de literatura. Perfiles educativos, 35(139), 128150. Recuperado de https://www.iisue.unam. $\mathrm{mx} /$ perfiles/articulo/2013-139-dificultades-paraimplementar-la-evaluacion-formativa-revisionde-literatura.pdf

Ministerio de Educación Nacional (2003). Manual de la Evaluación de Desempeño. Recuperado de https://www.mineducacion.gov. co/1621/articles-81030_archivo_pdf.pdf

Ministerio de Educación Nacional (2006). Estándares Básicos de Competencias en Lenguaje, Matemáticas, Ciencias y Ciudadanas Guía sobre lo que los estudiantes deben saber y saber hacer con lo que aprenden. Recuperado de https://www.mineducacion.gov.co/1621/ articles-340021_recurso_1.pdf

Ministerio de Educación Nacional (2008). Guía Metodológica, Evaluación Anual de desempeño Laboral. Recuperado de https://www. mineducacion.gov.co/1621/articles-169241_ archivo_pdf.pdf

Ministerio de Educación Nacional (2008b). Guía para el mejoramiento institucional: de la autoevaluación al plan de mejoramiento. Recuperado de https://www.mineducacion.gov. co/1621/articles-177745_archivo_pdf.pdf

Ministerio de Educación Nacional (2009) Organización del sistema educativo conceptos generales de la educación preescolar, básica y media. Recuperado de https://www. calidadeducativasm.com/wp-content/uploads/ guias_min_educacion/guia33_organizacion_ del_sistema_educativo.pdf 
Ministerio de Educación Nacional. (2014). Evaluación de competencias para el ascenso o reubicación de nivel salarial en el Escalafón de Profesionalización Docente de los docentes y directivos docentes regidos por el Decreto Ley 1278 de 2002. Documento Guía. Docente Media Ciencias Naturales y Educación Ambiental - Física. Recuperado de https://www. mineducacion.gov.co/1759/articles-342767_ recurso_nuevo_15.pdf

Ministerio de Educación Nacional. (2014b). Informe de resultados de la evaluación de competencias para el ascenso o la reubicación de nivel salarial en el escalafón de docentes y directivos docentes regidos por el decreto ley 1278 de 2002. Agregado nacional. Recuperado de https://www.mineducacion.gov.co/1621/ articles-244742_archivo_pdf_Informe_ resultados_evaluacion_competencias.pdf

Ministerio de Educación Nacional. (2017). La evaluación formativa y sus componentes para la construcción de una cultura de mejoramiento. Recuperado de http://aprende. colombiaaprende.edu.co/sites/default/files/ naspublic/La\%20 evaluaci\%C3\%B3n \% 20 formativa $\% 20 \mathrm{y} \% 20$ sus $\% 20$ componentes $\% 20$ para\%20la\%20construcci\%C3\%B3n\%20de\%20 una $\% 20$ cultura $\% 20 \mathrm{de} \% 20$ mejoramiento.pdf

Miñana, C. \& Rodríguez, J. G., (2003). La educación en el contexto neoliberal. En D. Restrepo (Ed.) La falacia neoliberal. Crítica y alternativas, (pp. 285-321). Bogotá: Universidad Nacional de Colombia.

Moreno, T. (2012). La evaluación de competencias en educación. Sinéctica, (39), 1-20. Recuperado de https://sinectica.iteso.mx/ index.php/SINECTICA/article/view/84

Moreno, T. (2016). Evaluación del aprendizaje y para el aprendizaje. Reinventar la evaluación en el aula. México: Universidad Autónoma Metropolitana.
Peña, L., Almuiñas, J. \& Galarza, J. (2018). La autoevaluación institucional con fines de mejora continua en las instituciones de Educación Superior. Universidad y Sociedad, 10(4), 18-24. Recuperado a partir de https://rus.ucf.edu.cu/ index.php/rus/article/view/972

Pereira, Z. (2011). Los diseños de método mixto en la investigación en educación: Una experiencia concreta. Revista Electrónica Educare, 15(1), 15-29. DOI: DOI: https://doi. org/10.15359/ree.15-1.2

Perkins, D. (1999). ¿Qué es la comprensión? En M. Stone (comp), La Enseñanza para la Comprensión. Vinculación entre la investigación y la práctica (pp. 69-94). Buenos Aires: Paidós

Prada, R., Hernández, C. \& Ramírez, P. (2020). Enseñanza del cálculo diferencial: un análisis de las dificultades en estudiantes universitarios. Bogotá: Ecoe Ediciones - Universidad Francisco de Paula Santander.

Romero, C. (2004). La escuela media en la sociedad del conocimiento Ideas y herramientas para la gestión educativa. Autoevaluación y planes de mejora. Buenos Aires: Ediciones Novedades Educativas.

Ricoeur, P. (1987). Tiempo y narración. Madrid, España: Siglo XXI.

Rueda, M. (2009). La evaluación del desempeño docente: consideraciones desde el enfoque por competencias. Revista Electrónica de Investigación Educativa, 11 (2). Recuperado de http://redie.uabc.mx/vol11no2/contenidorueda3.html

Ruedas, M.; Ríos, M. \& Nieves, F. (2009). Epistemología de la investigación cualitativa. Educere. Revista Venezolana de Educación, 13(46), 627-635. Recuperado de http://www. saber.ula.ve/handle/123456789/31247 
Salamanca-Meneses, X., \& HernándezSuárez, C. A. (2018). Enseñanza en ciencias: la investigación como estrategia pedagógica. Trilogía Ciencia Tecnología Sociedad, 10(19), 133-148. DOI: https://doi. org/10.22430/21457778.1025

Sandoval, C. (1997). Investigación cualitativa. Programa de Especialización en teoría, métodos y técnicas de investigación social. Bogotá: ICFES.

Santos, B. (2014). Competencias docentes para la enseñanza de ciencias naturales en una Institución Privada de nivel medio superior en el área Metropolitana de Monterrey, N.L. Recuperado de http://www.eumed.net/librosgratis/2014/1418/ciencias-naturales.htm

Schwerdt, G., \& Wuppermann, A. C. (2011). Is traditional teaching really all that bad? A withinstudent between-subject approach. Economics of Education Review, 30(2), 365-379. DOI: https:// doi.org/10.1016/j.econedurev.2010.11.005

Talanquer, V. (2015) La importancia de la evaluación formativa. Educación química, 26(3), 177-179. DOI: http://dx.doi.org/10.1016/j. eq.2015.05.001

Tamayo, J. (2017). La práctica pedagógica como categoría de análisis; acercamientos desde su construcción como objeto de investigación. Memoria electrónica del Congreso Nacional de Investigación Educativa, 3(3). Recuperado de http://www.comie.org.mx/congreso/ memoriaelectronica/v14/doc/1515.pdf

Taylor, S \& Bogdan, R. (1987). Introducción a los métodos cualitativos de investigación. La búsqueda de significados. Barcelona: Paidós

Tobón, S. (2005). Formación basada en competencias: Pensamiento complejo, diseño curricular y didáctica. Bogotá: Ecoe ediciones.
Touriñán López, J. M. (2020). Alcance de teoría de la educación en la carrera de pedagogía. Revista Boletín Redipe, 9(4), 25-89. https://doi.org/10.36260/rbr.v9i4.947

Trujillo, M. F. (2009). Educación, globalización y neoliberalismo: Ideas para una reflexión en el contexto colombiano. REDHECS, 6(4), 94-105. Recuperado de http://ojs.urbe.edu/index.php/ redhecs/article/view/71

Trujillo-Segoviano, J. (2014). El enfoque en competencias y la mejora de la educación. $R a$ Ximhai, 10(5), 307-322. Recuperado de https:// www.redalyc.org/pdf/461/46132134026.pdf

Veloza, R., \& Hernández, C. (2018). Valoración de las estrategias adoptadas por docentes en la enseñanza de la ciencia desde la perspectiva de los estudiantes de educación básica. Ánfora, 25(45), 43-69. DOI: https://doi.org/10.30854/anf. v25.n45.2018.512

Zabala, A. \& Arnau, L. (2007). La enseñanza de las competencias. Aula de innovación educativa, 161, 40-46. Recuperado de https:// www.grao.com/es/producto/la-ensenanza-delas-competencias-17238

Zuluaga, O. L. (1999). Pedagogía e historia: la historicidad de la pedagogía. La enseñanza, un objeto de saber. Bogotá: Siglo del Hombre Editores, Anthropos, Editorial Universidad de Antioquia 